Communications in Physics, Vol. 24, No. 2 (2014), pp. 135-140

\title{
ELECTRICAL TRANSPORT PROPERTIES OF Ni-Cr BINARY ALLOYS
}

\author{
P. H. SUTHAR \\ Department of Physics, C U Shah Science College, \\ Ahmedabad 380 014, Gujarat, India
}

B. Y. THAKORE

Department of Physics, Sardar Patel University, Vallabh Vidyanagar 388 120, Gujarat, India

P. N. GAJJAR

Department of Physics, University School of Sciences, Gujarat University, Ahmedabad 380 009, Gujarat, India

E-mail: sutharpunit@rediffmail.com

Received 09 December 2013

Accepted for publication 13 May 2014

\begin{abstract}
Electrical transport properties viz. electrical resistivity and thermal conductivity of Ni-Cr binary alloys are determined by our recognized single parametric potential model in a wide range of $\mathrm{Cr}$ concentration. In this work, screening functions of Ichimaru and Utsumi, Farid et al. and Sarkar et al. are employed along with the Hartree and Taylor functions to study the relative influence of the exchange and correlation effect. The Ni-Cr liquid alloys are studied as a function of their composition at three different temperatures $(T=800 \mathrm{~K}, 1000 \mathrm{~K}, 1200 \mathrm{~K})$. The present finding of electrical resistivity agrees within 2-12\% with experimental findings when the $H$ dielectric function is employed.
\end{abstract}

Keywords: Liquid alloys, Electrical resistivity, Pseudopotential, Exchange and correlation effects.

\section{INTRODUCTION}

The electrical transport properties of liquid metals and their alloys are of immense important theoretically as well as experimentally because of increasingly manifold interests of not only physicists but also chemists. During last three decades numbers of efforts are made to study electrical transport properties of liquid metals and their alloys. The Faber-Ziman [1-4] model remain successful in predictive the electrical transport properties of binary. Thakore et al. [3-4] have successfully reported that the structures and electrical transport properties using Ashcroft and our well established local model potential for different binary alloys $\mathrm{Al}-\mathrm{Cu}$ and $\mathrm{Sn}-\mathrm{Sb}$, respectively. Very recently, we carefully computed the electrical resistivity and thermal conductivity of liquid $\mathrm{Ni}-\mathrm{Cr}$ at $1400 \mathrm{~K}$ [5]. We have also reported that electrical transport properties using the nearly free electron theory developed by Faber and Ziman formalism, along with a well established potential model [3-5].

(C)2014 Vietnam Academy of Science and Technology 
As, it is known that Ni-Cr transition metal (TM-alloy) alloys based on iron-subgroup metal take up a special interest in modern technology [6]. Since, they are important starting material for many industrial applications and manufacture of surgical implant. Glagoleva et al [6] have measured experimentally electrical resistivity and thermal conductivity at various temperatures. They have found that the electrical transport properties more sensitive at high temperature. In the present work, we report the results of our theoretical study of electrical transport properties of liquid alloy Ni-Cr. The objective of the paper is of two fold: one is to study the influence of the screening functions and the second is to study the temperature effect. For these, five different form of the local field correction functions are used and three different temperatures $\mathrm{T}=800 \mathrm{~K}, 1000 \mathrm{~K}$, $1200 \mathrm{~K}$ are considered.

\section{COMPUTATIONAL METHOD}

The electrical resistivity for binary alloys in Faber and Ziman $[1,2]$ formulation is given as,

$$
\rho=\frac{3 \pi \Omega m^{2}}{4 e^{2} \hbar k_{F}^{6}} \int_{0}^{2 k_{F}} S(\mathbf{q})|V(q)|^{2} \mathbf{q}^{3} \theta\left(2 k_{F}-\mathbf{q}\right) d q,
$$

where, $n$ is the electron density related to the Fermi wave number, $\theta$ the unit step function that cuts off the q-integration at $2 k_{F}$ corresponding to a perfectly sharp Fermi surface, $S(q)$ the structure factor and $V(q)$ the screened ion pseudopotential form factor. To incorporate the electron-ion interaction we have used Gajjar's recognized model potential [5, 7]. In momentum space the unscreened bare-ion form factor is given by [5, 7],

$$
V_{b}(q)=\frac{-4 \pi Z e^{2}}{\Omega \mathbf{q}^{2}}\left[\cos \left(q r_{c}\right)-\frac{\mathbf{q}^{2} r_{c}^{2}}{1+\mathbf{q}^{2} r_{c}^{2}}\right] .
$$

Here $Z, \Omega, q, e$ and $r_{c}$ are the valency, atomic volume, wave vector, charge of the electron and the parameter of the potential, respectively. The approach of Faber and Ziman [1-2] is used to study the concentration dependence of the electrical resistivity of liquid binary mixture [6]. In the present computation of electrical transport properties of liquid binary alloys, the local field correction functions due to Hartree (H) [8], Taylor (T) [9], Ichimaru and Utsumi (IU) [10], Farid et al. (F) [11] and Sarkar et al. (S) [12] are employed for the investigation of exchange and correction effects. Hartree function does not involve any kind of exchange and correlation effects. While, other four functions are satisfied compressibility sum rule and they have been used earlier by many researchers to analyse various physical, electrical and magnetic properties of liquid metals and their alloys [3-6]. The parameters of the model potential for pure $\mathrm{Ni}$ and $\mathrm{Cr}$ have been determined using $q=q_{0}$ condition. Hence, the present study does not include any kind of fitting process. On the rearrangements of the various constants, one can write the formula (1) for the electrical resistivity of the binary alloys in the following form:

$$
\rho=\frac{12.81 \Omega}{k_{F}^{6}} \int_{0}^{2 k_{F}} \mathbf{q}^{3} S(\mathbf{q}) d q
$$

with

$$
S(q)=(1-x) S_{11}(q) V_{1}^{2}(q)+2[x(1-x)]^{1 / 2} S_{12}(q) V_{1}(q) V_{2}(q)+x S_{22}(q) V_{2}^{2}(q) .
$$


Here, $V_{1}(q)$ and $V_{2}(q)$ are denoted as the screened form factors for elements $A$ and $B, S_{i j}(q)$ are the Ashcroft-Langreth partial structure factors of the binary metal complexes [6], $x$ is the concentration of a second metallic component of the $A_{1-x} B_{x}$ mixture.

When the temperature gradient applied to metal, the conduction electron will carry a heat. Even though an electric current is prevented from flowing and that indeed they are responsible for the major part of the thermal conductivity, the expression for conductivity is written as $[3,4]$

$$
\sigma_{L}=\frac{\pi^{2} k_{B}^{2} T}{3 e^{2} \rho_{L}} .
$$

Here, $e, E_{F}, T$, and $k_{B}$ are the electronic charge, Fermi energy, temperature in Kelvin and the Boltzmann's constant.

\section{RESULTS AND DISCUSSIONS}

The input parameters used in the present computations are presented in Table 1. Moreover, packing fraction of liquid binary alloys is kept at 0.45 . The concentration dependence of the electrical resistivity at three different temperatures are examined by varying the $\mathrm{Cr}$ concentration from $x=0$ to $x=1.0(x=0,0.05,0.1,0.2,0.3,0.5,0.65,0.9,0.95,1.0)$.

Table 1. Input parameters used in the calculation

\begin{tabular}{|c|c|c|c|c|c|c|}
\hline \multirow{2}{*}{ Input } & \multicolumn{2}{|c|}{$\mathrm{T}=800 \mathrm{~K}$} & \multicolumn{2}{c||}{$\mathrm{T}=1000 \mathrm{~K}$} & \multicolumn{2}{c|}{$\mathrm{T}=1200 \mathrm{~K}$} \\
\hline & $\mathrm{Ni}$ & $\mathrm{Cr}$ & $\mathrm{Ni}$ & $\mathrm{Cr}$ & $\mathrm{Ni}$ & $\mathrm{Cr}$ \\
\hline$\sigma(\mathrm{au})$ & 4.05139 & 4.16 & 4.07713 & 4.185 & 4.1035 & 4.2056 \\
\hline$\Omega\left(\mathrm{au}^{3}\right)$ & 77.3345 & 84.054 & 78.8589 & 85.288 & 80.4014 & 86.55 \\
\hline $\mathrm{r}_{c}(\mathrm{au})$ & 1.348 & 1.3858 & 1.3570 & 1.3930 & 1.3655 & 1.3990 \\
\hline
\end{tabular}

Figs. 1-3 represent the computed values of electrical resistivity for Ni-Cr alloy at $800 \mathrm{~K}$, $1000 \mathrm{~K}$ and $1200 \mathrm{~K}$, respectively. From the Figs. 1-3 it is seen that, among five employed local field correction functions, $H$ gives the minimum numerical value of the electrical resistivity, while the local field correction functions due to $F$ gives the maximum value. In the present findings of the electrical resistivity of Ni-Cr binary alloy due to $T, I U$ and $S$ local field correction functions are laying between $H$ and $F$ functions. The results due to $\mathrm{H}$ and $\mathrm{S}$ functions show comparable agreement with experimental results among all five local field correction functions. At $800 \mathrm{~K}$ temperature, the deviation in the electrical resistivity with experimental values is $10.58-3.77 \%$, 76.86-56.17\%, 83.60-63.76\%, 85.83-66.0\% and 41.27-21.92\% of $H, T, I U, F$ and $S$ functions, respectively. At $1000 \mathrm{~K}$, such deviation is found as 5.24-2.31\%, 59.23-54.04, 66.69-59.28\%, 69.0$62.0 \%$ and $25.0-28.15 \%$ of $H, T, I U, \mathrm{~F}$ and $\mathrm{S}$ functions, respectively. For $1200 \mathrm{~K}$ temperature, such deviations are $12.98-2.5 \%, 59.39-41.72 \%, 66.89-46.88 \%, 69.24-49.03 \%$ and $12.90-24.015 \%$ from experimental data is observed for $H, T, I U, F$ and $S$ functions, respectively. From Fig. 13 , it is also seen that as the temperature of binary liquid increases, the electrical resistivity also increases favorite metallic behavior of binary mixture $\mathrm{Ni}-\mathrm{Cr}$ over the full range of concentration. 


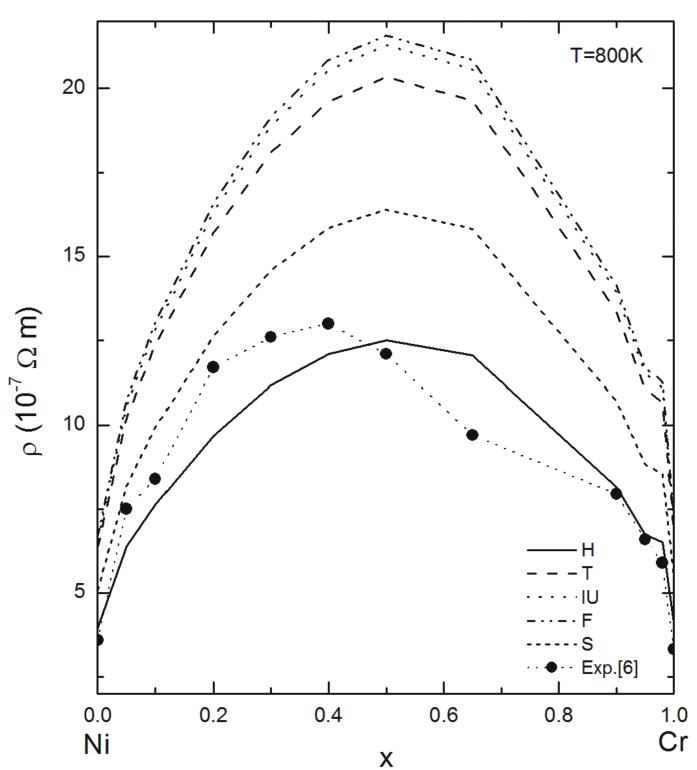

Fig. 1. Electrical resistivity of $\mathrm{Ni}_{1-x} \mathrm{Cr}_{x}$ alloy at $T=800 \mathrm{~K}$

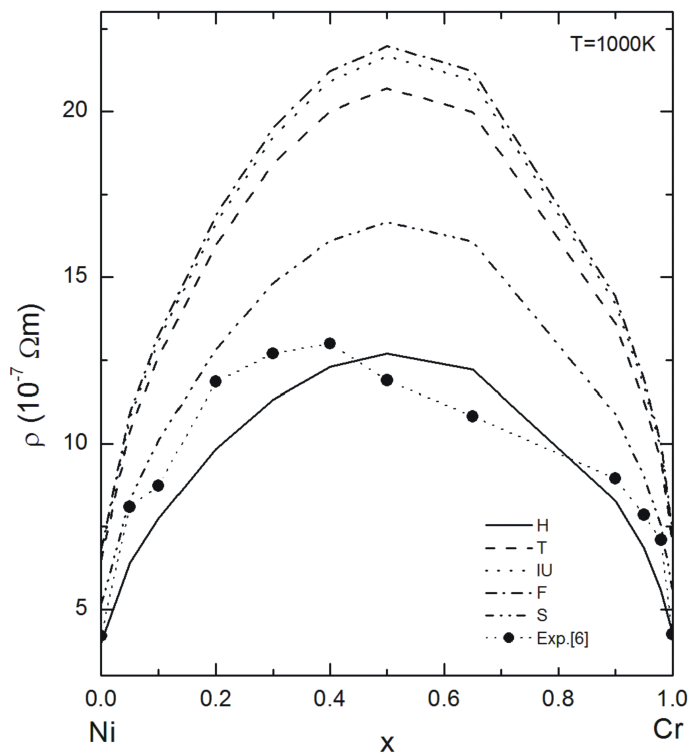

Fig. 2. Electrical resistivity of $\mathrm{Ni}_{1-x} \mathrm{Cr}_{x}$ alloy at $T=1000 \mathrm{~K}$

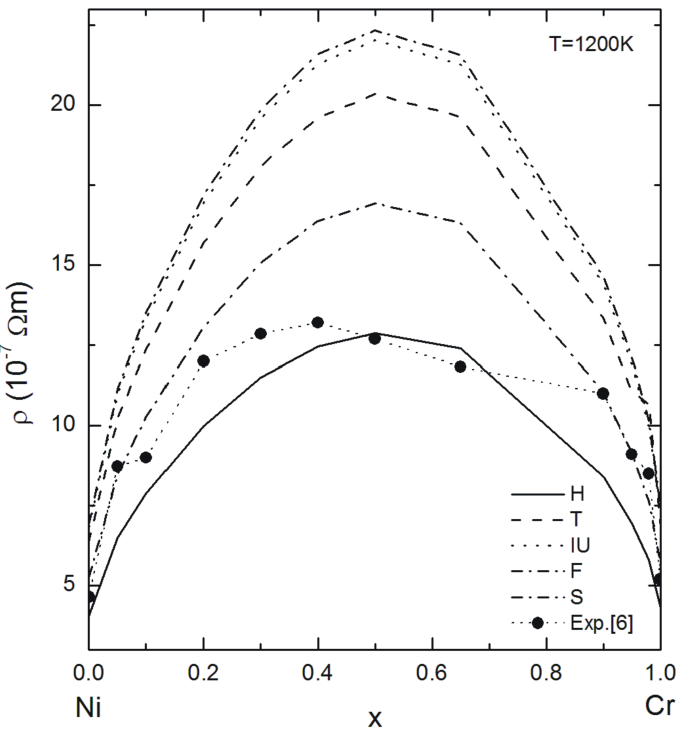

Fig. 3. Electrical resistivity of $\mathrm{Ni}_{1-x} \mathrm{Cr}_{x}$ alloy at $T=1200 \mathrm{~K}$

In Figs. 4-6, thermal conductivity of binary Ni-Cr alloy is shown along with the experimental results [6]. For $0<x<1$, the thermal conductivity of binary mixture $\mathrm{Ni}_{1-x} \mathrm{Cr}_{x}$ is found to be lower than the thermal conductivity of pure components $\mathrm{Ni}, \mathrm{Cr}$. Moreover, $\mathrm{H}$ gives the maximum value of the thermal conductivity; while the screening functions $\mathrm{F}$ gives the minimum value the conductivity of the $\mathrm{Ni}-\mathrm{Cr}$ alloy in comparison to other screening functions. 


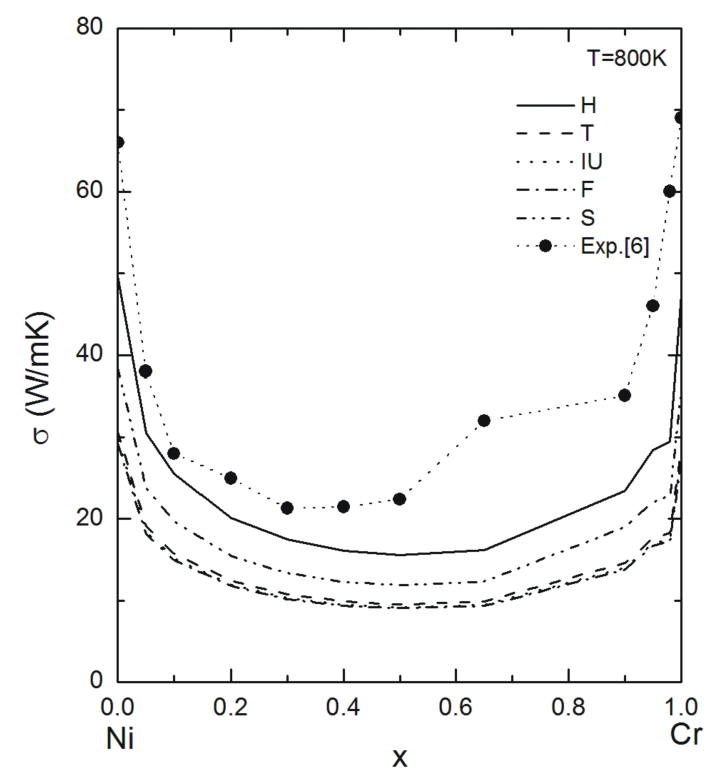

Fig. 4. Thermal conductivity of $\mathrm{Ni}_{1-x} \mathrm{Cr}_{x}$ alloy at $T=800 \mathrm{~K}$

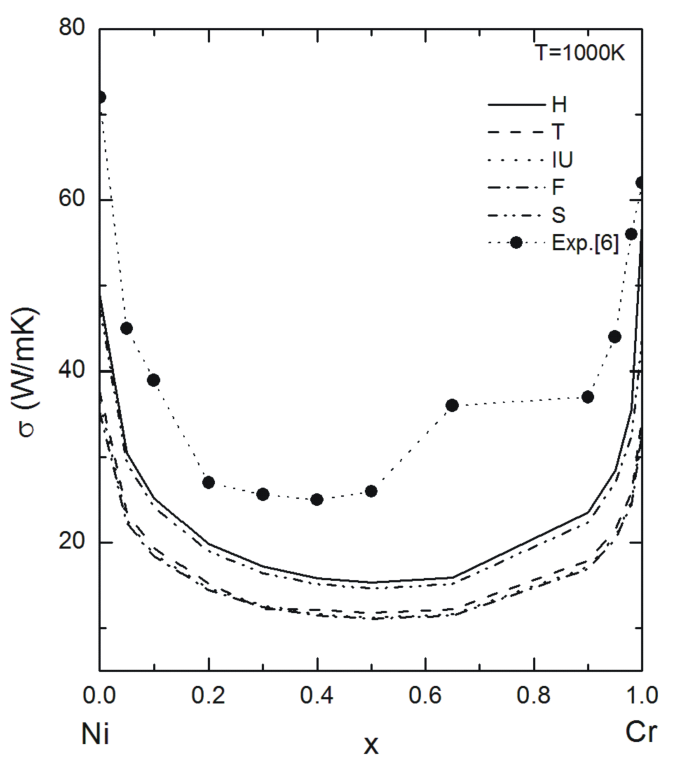

Fig. 5. Thermal conductivity of $\mathrm{Ni}_{1-x} \mathrm{Cr}_{x}$ alloy at $T=1000 \mathrm{~K}$

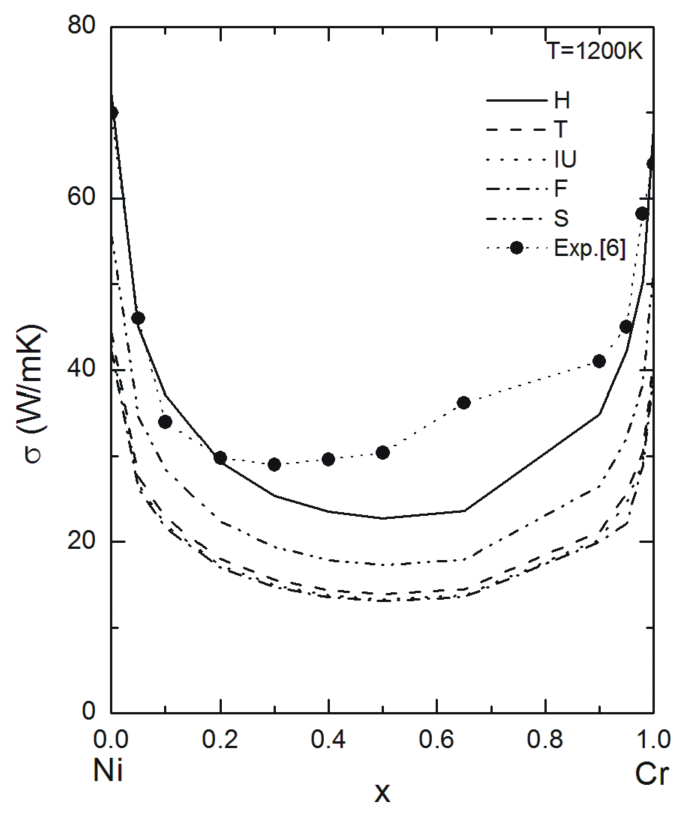

Fig. 6. Thermal conductivity of $\mathrm{Ni}_{1-x} \mathrm{Cr}_{x}$ alloy at $T=1200 \mathrm{~K}$

In the case of thermal conductivity, deviation with respect to experimental data is found in the $H, T, I U, F$ and $S$ screening functions and the values are $24.71-22.02 \%$, for $\mathrm{H}$ screening function, 55.30-53.50\% for $T$ screening function, 56.99-55.10\% for $I U$ screening function, 57.32$55.80 \%$ for $F$ screening function and $44.13-41.84 \%$ for $S$ screening function for Ni-Cr alloy at 
$T=800 \mathrm{~K}$. Similarly, at $1000 \mathrm{~K}$, such deviations found are $38.52 \%-20.37 \%$, 52.88-47.62\%, 55$49.33 \%, 55.6-50.89 \%$ and $41.44-34.20 \%$ for $H, T, I U, F$ and $S$ functions, respectively. At $1200 \mathrm{~K}$ temperature, such deviations are $23.69-3.37 \%, 52.03-36.33 \%, 54.17-38.77 \%, 54.82-39.64 \%$ and $40.38-20.32 \%$ for H, T, IU, F and S functions, respectively.

The computed values of the electrical transport properties viz. the electrical resistivity and thermal conductivity is found to be quite sensitive to the selection of the appropriate screening functions.

The screening function due to IU and F shows the peak around the $q \approx 2 k_{f}$, so that the present results of electrical resistivity give higher value than the other screening function. The $\mathrm{S}$ function is obtained very mild peak around the $q \approx 2 k_{f}$. Hence, it can be said that the $\mathrm{S}$ function gives very good results compared with the IU and F functions. Overall, the results of electrical transport properties computed here for $\mathrm{Ni}-\mathrm{Cr}$ binary mixture suggests that the proper choice of exchange and correlation function is essential for the study of the electrical transport properties of binary system.

\section{CONCLUSION}

At last we conclude that electrical transport properties of $\mathrm{Ni}_{1-x}-\mathrm{Cr}_{x}$ alloys due to $\mathrm{H}$ and $S$ local field correction functions are found to be in good agreement with experimental results. The $S$ function, which satisfies the compressibility sum rule in the long wavelength limit more accurately as compared to $T, I U$ and $F$ functions and hence gives better results. Thus, the present computation confirms not only applicability of our model potential for the study of the electric resistivity and thermal conductivity of binary alloys, but it also establishes the use of more prominent dielectric functions in the study of electrical transport properties of such liquid binary mixtures.

\section{ACKNOWLEDGMENTS}

The authors BYT is thankful to UGC for providing financial support under major research project. The authors PNG acknowledge the computer facilities developed under DST-FIST level-I and financial assistant received under DRS-SAP-I.

\section{REFERENCES}

[1] T. E. Faber and J. M. Ziman, Philos. Mag. 11 (1965) 153.

[2] J. M. Ziman, Philos. Mag. 6 (1961)1013.

[3] B Y Thakore, P H Suthar, S G Khambholja and A R Jani, AIP Conference Proceedings 1249 (2010) 170.

[4] B Y Thakore, S G Khambholja, P H Suthar and A R Jani, AIP Conference Proceedings 1249 (2010) 194.

[5] P H Suthar, S G Khambholja, B T Thakore, P N Gajjar and A R Jani, AIP Conference Proceedings 1349 (2011) 945.

[6] Yu. V. Glagoleu, N. B. Pushareva, Yu. E. Lapshora, O. V. Sadyreva, V. F. Polev, V. I. Gorbatov, S. G. Talutls and I. G. Korshnuov, The Phys. of Metal and Metallography 102 (2006) 48.

[7] A. R. Jani, P. N. Gajjar and H K Patel, Phys. Stat. Sol. B 169 (1991) K 105.

[8] W. A. Harrison, Pseudopotential in the Theory of Metal, W A Benamin Inc, New York (1966).

[9] R. Taylor, J. Phys. F8 (1981)1699.

[10] S. Ichimaru and K. Utsumi, Phys. Rev. 24 (1981) 3220.

[11] B. Farid, V. Heine, G.E. Engel, I. J. Robertson, Phys. Rev. B 48 (1993) 11602.

[12] A. Sarkar, D. S. Sen, H. D. Roy, Mod. Phys. Lett. 12 (1998) 639. 